\title{
Compressible and stretchable magnetoelectric sensors based on liquid metals for high-sensitive, self-powered respiratory monitoring
}

Xuan Zhang, ${ }^{a, c}$ Jingwei Ai, ${ }^{b}$ Ruiping Zou, ${ }^{c}$ Bin Su*a

${ }^{a}$ State Key Laboratory of Material Processing and Die \& Mould Technology, School of Materials

Science and Engineering, Huazhong University of Science and Technology, Wuhan 430074, Hubei, P. R. China,

${ }^{b}$ State Key Laboratory of Advanced Electromagnetic Engineering and Technology, School of Electrical and Electronic Engineering, Huazhong University of Science and Technology, Wuhan 430074, Hubei, China,

${ }^{c}$ ARC Hub for Computational Particle Technology, Department of Chemical Engineering, Monash University, Clayton VIC 3800, Australia.

* To whom correspondence should be addressed: B. Su. (subin@hust.edu.cn) 
(a)

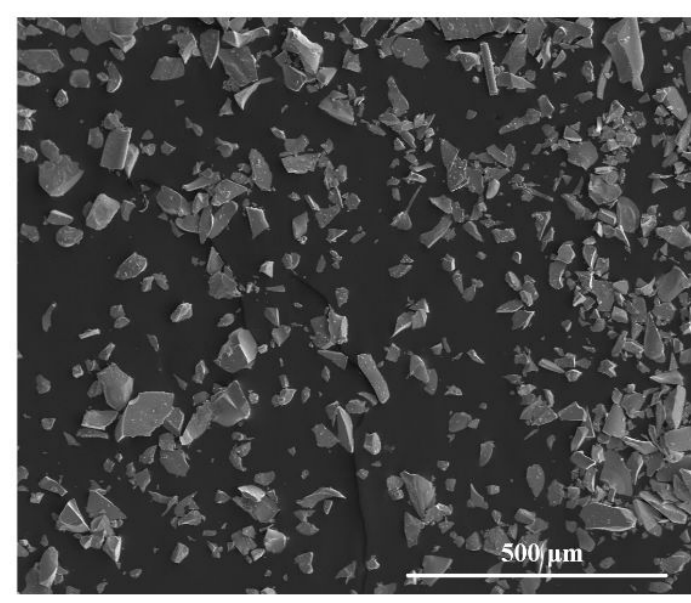

(b)

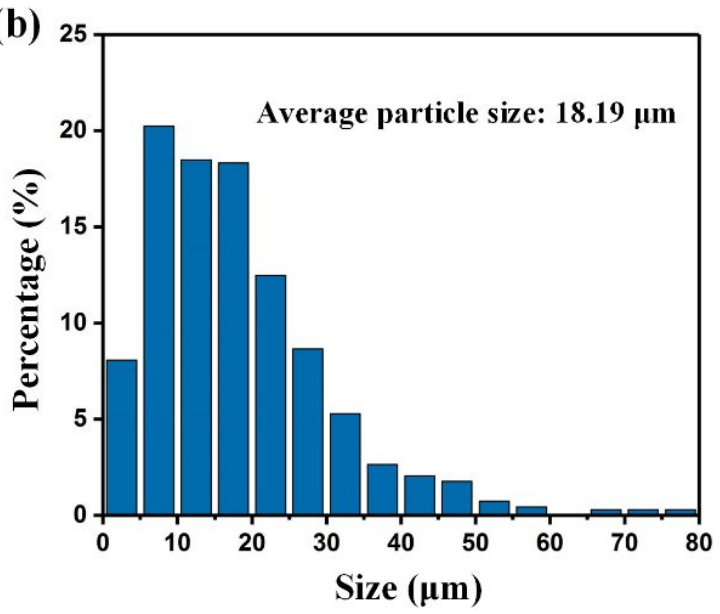

Figure S1. Particle characterization of 400 -mesh $\mathrm{Nd}_{2} \mathrm{Fe}_{14} \mathrm{~B}$ powders. (a) The ESEM image of $\mathrm{Nd}_{2} \mathrm{Fe}_{14} \mathrm{~B}$ powders that have been not experienced a magnetization process. (b) Statistical analysis on particle distribution of $\mathrm{Nd}_{2} \mathrm{Fe}_{14} \mathrm{~B}$ powders, with an average particle size of $18.19 \mu \mathrm{m}$.

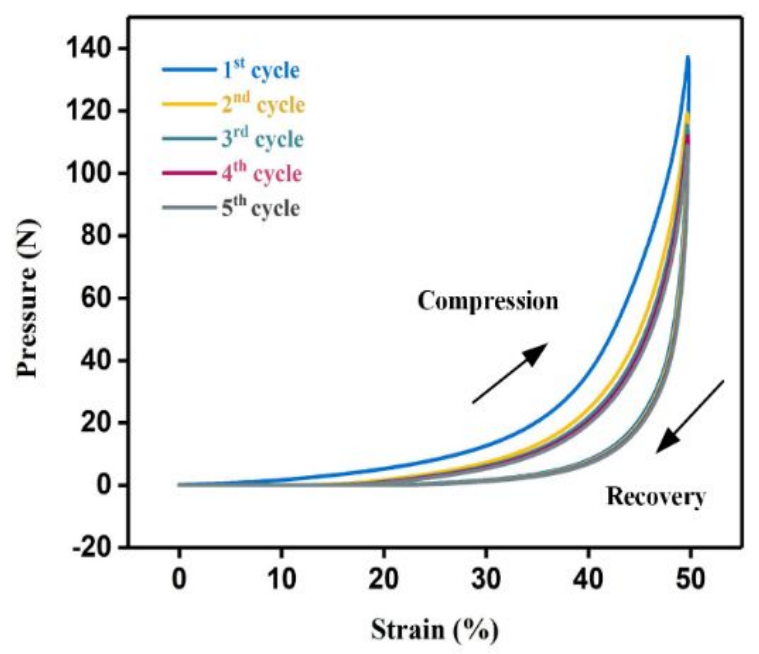

Figure S2. The correlation loading force and strain for the magnetic film with a $6 \times 6$ pyramid-bulge array (pyramid size: $2 \times 2 \times 3 \mathrm{~mm}$ ) during five continuous compression and recovery cycles. 
(a)

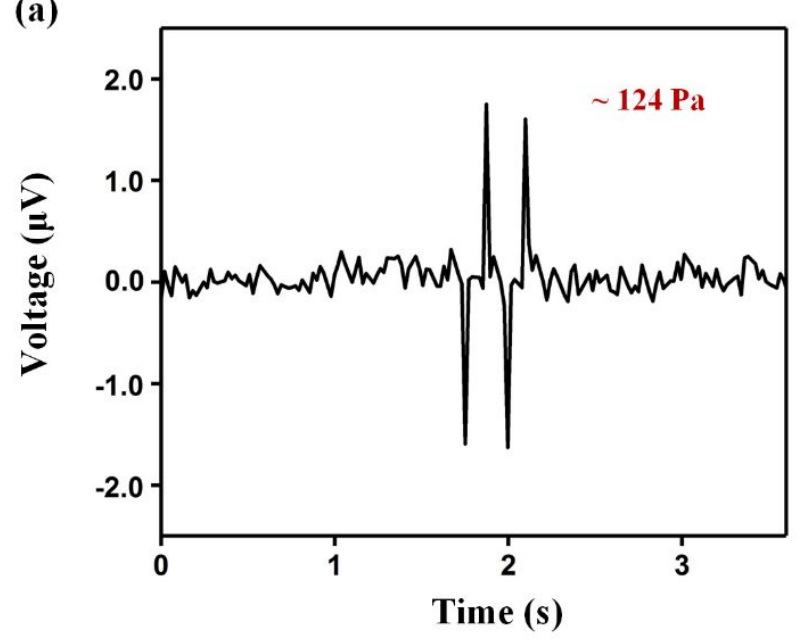

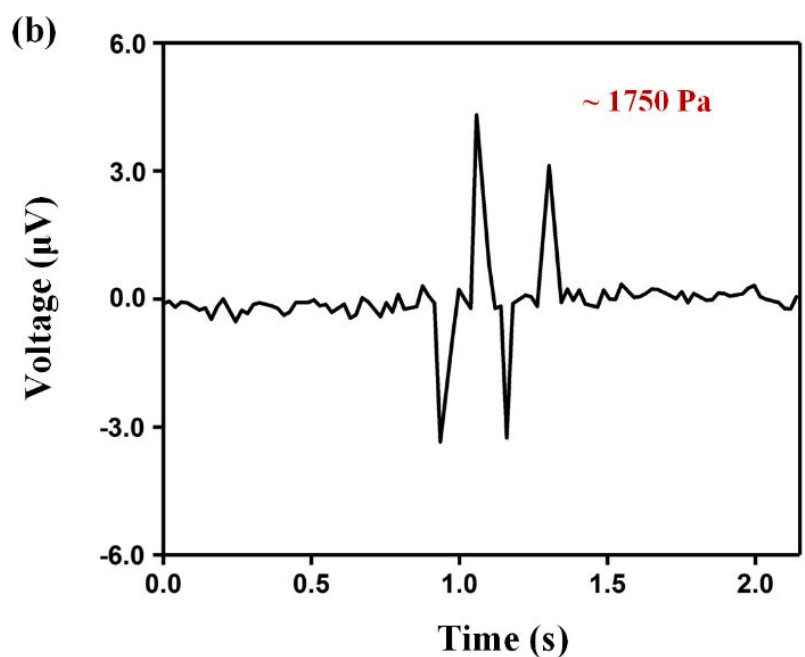

Figure S3. The minimum response to (a) pressure and (b) tension of the CSMS, as low as around 124 $\mathrm{Pa}$ and $1750 \mathrm{~Pa}$, respectively.

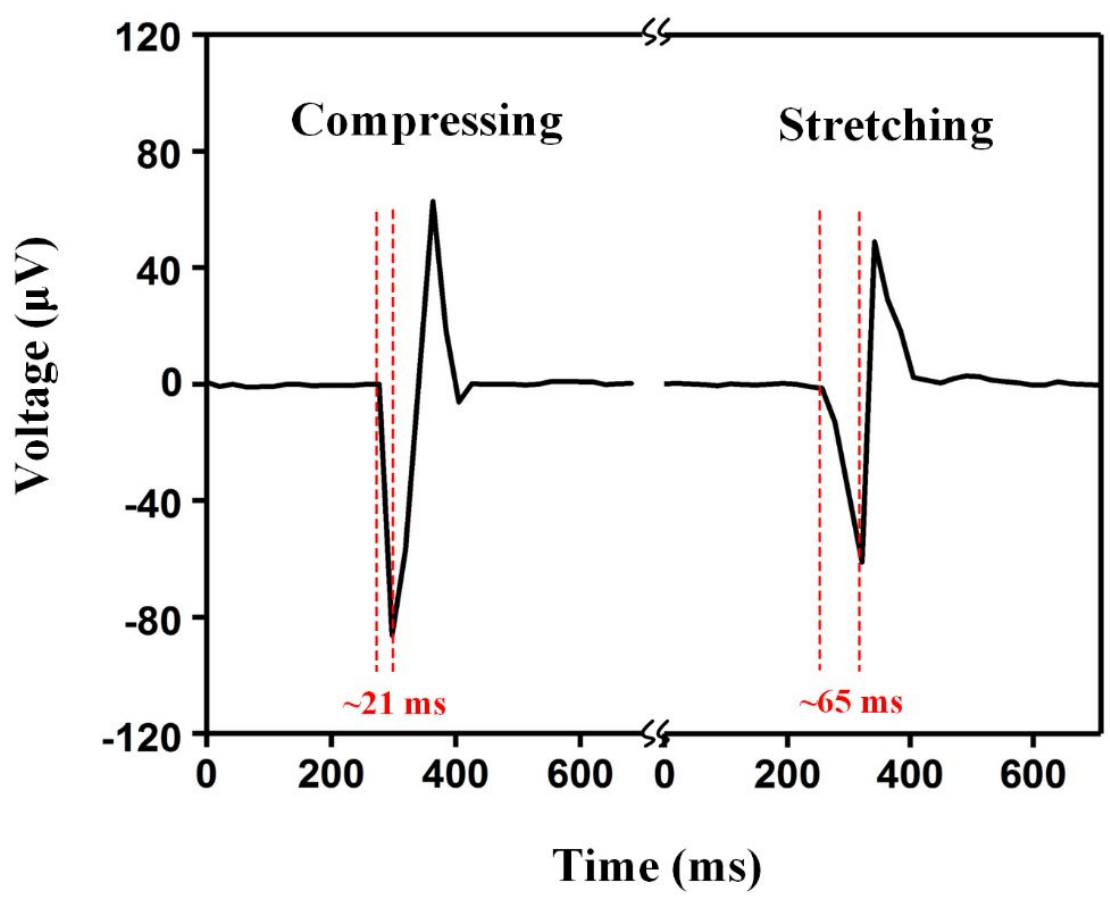

Figure S4. Instant response of the CSMS by a finger tapping or a gentle stretching. 


\section{Note S1. The unary equivalent modelling of magnetic-powders/polymer mixture for CSMSs}

The ANSYS MAXWELL finite element analysis software was utilized to conduct 3D modeling of magnetic films $\left(\mathrm{Nd}_{2} \mathrm{Fe}_{14} \mathrm{~B}\right.$ particles/Ecoflex mixture) integrated with liquid-metal patterns. The 3D simulation modeling employed a 2D static magnetic field solver added with a vertical Z-axis. Each magnetic particle was set as a $18 \mu \mathrm{m} \times 18 \mu \mathrm{m} \times 18 \mu \mathrm{m}$ cube that was magnetized in the direction of positive $\mathrm{Z}$-axis (the top and bottom for $\mathrm{N}$ and $\mathrm{S}$ poles, respectively) according to $\mathrm{Nd}_{2} \mathrm{Fe}_{14} \mathrm{~B}$ parameters . In this case, the magnetic coercivity of $\mathrm{Hc}$ was set as $-626.67 \mathrm{KA} / \mathrm{m}$, as well as residual magnetism $\mathrm{Br}$ of $0.7875 \mathrm{~T}$.

Traditional simulation can only calculate the distribution of magnetic intensity around a few magnetic bulks. The Simulation of magnetic intensity distribution for multitudinous magnetic powders requires considerable calculation capacities, which cannot be treated by current computer systems. Accordingly, an equivalent model, by treating binary magnetic-powders/polymer as a unary magnet, has been undertaken in Maxwell numerical simulation to simplify the calculation. Before building the simulation modeling, several ideal conditions should be taken into consideration as below:

1) The gap between neighboring magnetic powders is uniform and equal;

2) The deflection of magnetic powders is not considered due to the magnetic interaction force between magnetic powders.

3) Magnetizing the magnetic powders along the same direction (Z-axis).

A $\mathrm{Nd}_{2} \mathrm{Fe}_{14} \mathrm{~B}$ magnetic-particle matrix inside the Ecolflex polymer (binary system, see Figure S4a) and an individual magnet in the same size and shape (equivalent unary model, see Figure S4b) 
were constructed, respectively. The magnetic particle content in the binary system is $70 \mathrm{wt} \%$, namely equal to $\sim 30$ vol\%. In the unary equivalent model, only the residual magnetism $\mathrm{Br}$ changed while other parameters were consistent with those of $\mathrm{Nd}_{2} \mathrm{Fe}_{14} \mathrm{~B}$ magnetic-particle matrix (including permeability and magnetic coercivity). The calculation results of the models in the ANSYS MAXWELL software are shown in the Figure S4c,d.

To obtain equivalent distributions of magnetic intensity of binary magnetic-particle matrix inside the polymer (Figure S4c) and the unary model (Figure S4d), Br of the unary magnet model should be declined to $0.2359 \mathrm{~T}$ that is about 0.3 times of the $\mathrm{Nd}_{2} \mathrm{Fe}_{14} \mathrm{~B}$ blocks $(\mathrm{Br}=0.7875 \mathrm{~T})$. As a result, these two mapping distributions for magnetic density of these two models along the $\mathrm{Z}$ axis (black line) can match well (Figure S4f), which is a quantitative comparison for these two models. The curve of binary magnetic system (red line) is in accord with the curve of the equivalent unary model (blue line). These results show that binary magnetic-powders/polymer mixture (mass ratio of 7/3) can be simplified as a unary magnet, by decreasing the residual magnetism $\mathrm{Br}$ of the unary magnet model to 0.3 times of that of the $\mathrm{Nd}_{2} \mathrm{Fe}_{14} \mathrm{~B}$ blocks, in the ANSYS MAXWELL finite element analysis. 
(a)

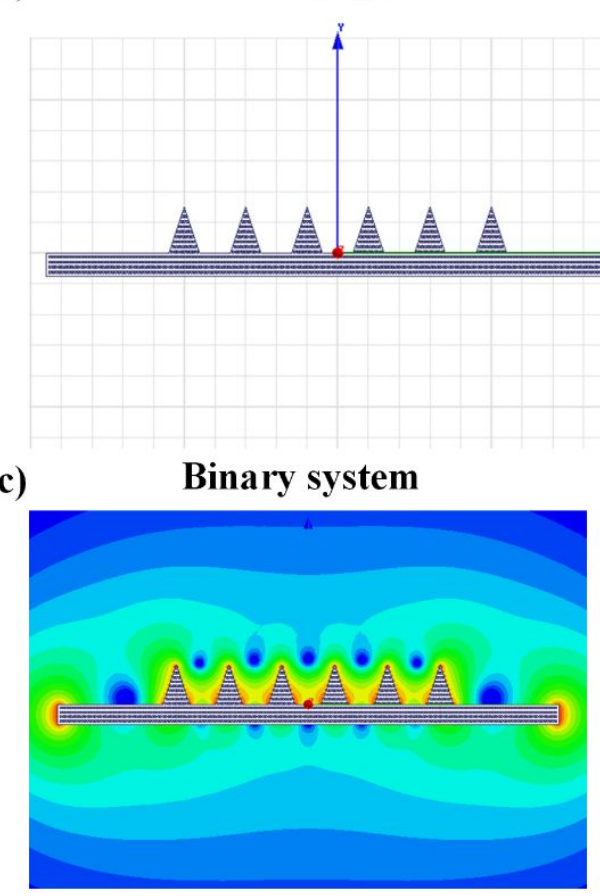

(b)

Unary system

\section{Equivalent to}

(d)

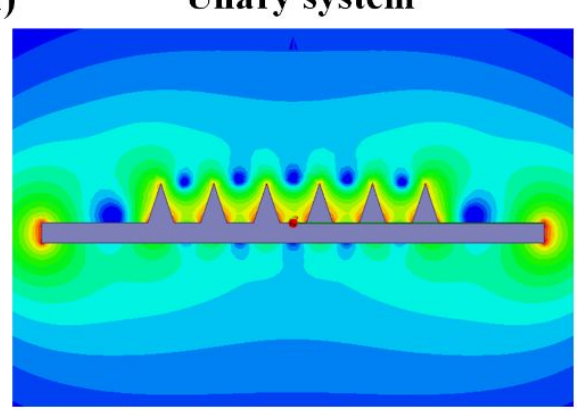

(e)

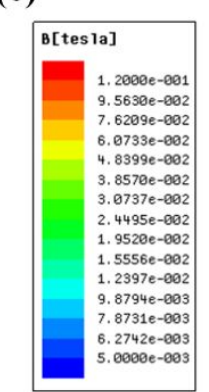

(f)

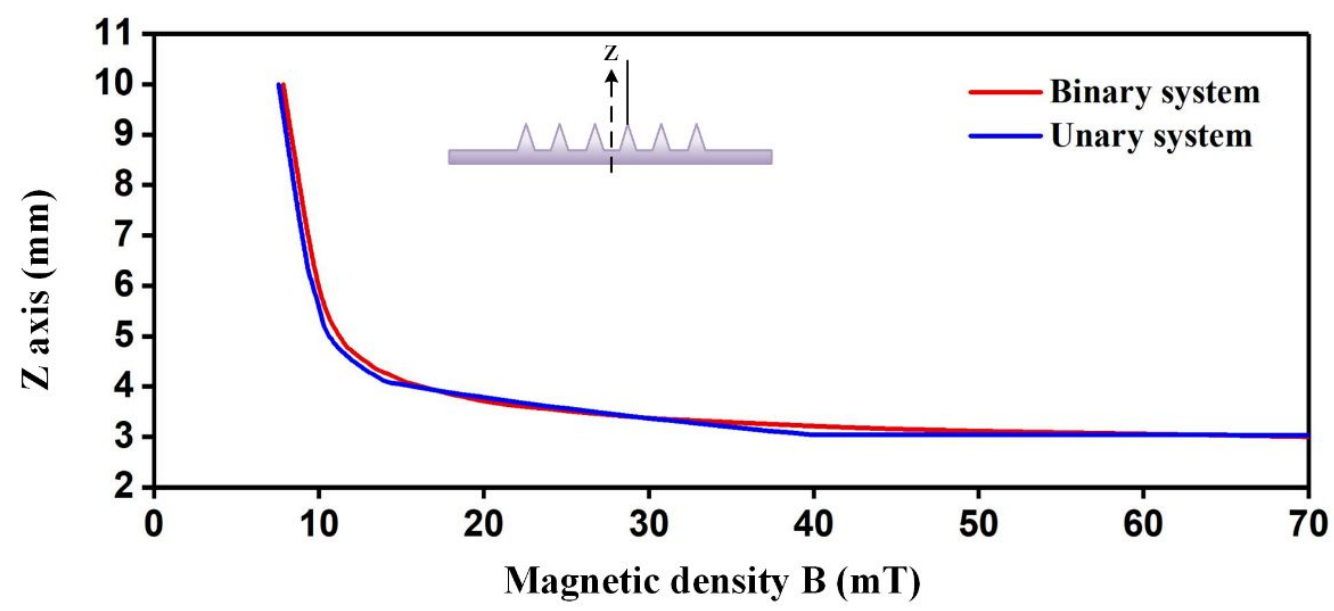

Figure S5. Calculation models of (a) binary magnetic powders \& Ecoflex mixture and equivalent (b) unary magnet unit. The mass ratio of magnetic-powders/Ecoflex is 7/3. (c) and (d) are 2D calculated distribution (y-z plane) of magnetic intensity of (a) and (b), respectively, as well as (e) their color bar representing the magnetic intensity. (f) The dependence of magnetic intensity on the distance along $\mathrm{Z}$ axis (black line) of the binary (red) and unary (blue) systems. The two lines are nearly in accord with each other. 


\section{Note S2. Detailed calculation of magnetic flux for CSMSs before/after deformation}

According to the simplified equivalent model in Note S1, the calculation of magnetic flux changes of CSMSs compressed or stretched can be carried out. Thus, the magnetic coercivity (Hc) was set as $-187.722 \mathrm{KA} / \mathrm{m}$ and the residual magnetism $(\mathrm{Br})$ was set as $0.2359 \mathrm{~T}$.

In simulation modeling, the Omega-shaped liquid metal rings involved in experiments were simplified as concentric rings in the same diameters as following conditions:

1) Four-cycle Omega-shaped liquid metals are equivalent to the same number of rings with diameter ranging from $14 \mathrm{~mm}, 18 \mathrm{~mm}, 22 \mathrm{~mm}$ and $26 \mathrm{~mm}$;

2) The equivalent rings are concentric rings and keep at the same plane;

3) The liquid-metal rings are in an infinite vacuum.

Based on Faraday's law of electromagnetic induction:

$$
E=-\frac{\Delta \Phi}{\Delta t}
$$

where $\mathrm{E}$ is the induced electromotive force, $\Delta \Phi$ is the change of total magnetic flux through fourcycle liquid metal rings, and $\Delta t$ is the response time of deformation (compression/stretch).

$$
\begin{aligned}
& \Delta \Phi=\Phi_{\text {after }}-\Phi_{\text {before }} \\
& \Delta t=t_{\text {after }}-t_{\text {before }}
\end{aligned}
$$

where $\Phi_{\text {after }}$ is the total magnetic flux of the sample after compressing/stretching, and $\Phi_{\text {before }}$ is the total magnetic flux of the sample in the original state. Similarly, $t_{\text {after }}$ is the moment after the sample was compressed/stretched, and $t_{\text {before }}$ is the moment when the sample just begins to compress/stretch.

The total magnetic flux can be calculated as follows: 


$$
\begin{array}{r}
\Phi=\oint_{S} \vec{B} \cdot \vec{S} d s \\
\Phi_{\text {after }}=\sum_{i=1}^{4} \Phi_{i} \\
\Phi_{\text {before }}=\sum_{i=1}^{4} \Phi_{i}
\end{array}
$$

Where $\vec{B}$ and $\vec{S}$ are the vector form of magnetic intensity and area of the liquid-metal concentric rings, respectively. And $\Phi_{\text {after }}$ and $\Phi_{\text {before }}$ could be calculated in the field calculator of postprocessing in ANSYS MAXWELL software. The calculated results for a compression/stretch have been shown in Table 1 in the manuscript. 
(a)

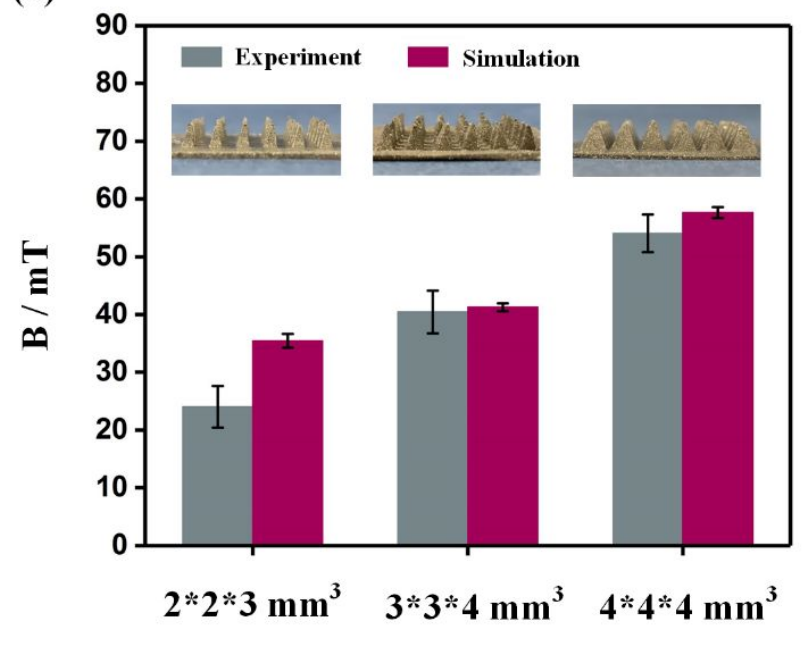

(c)

$2 * 2 * 3 \mathrm{~mm}^{3}$

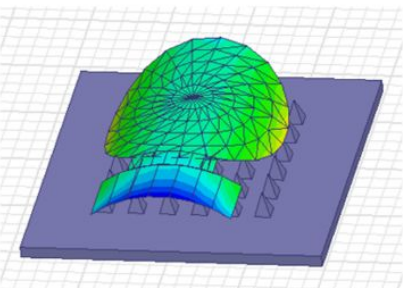

Compression

n Recovery

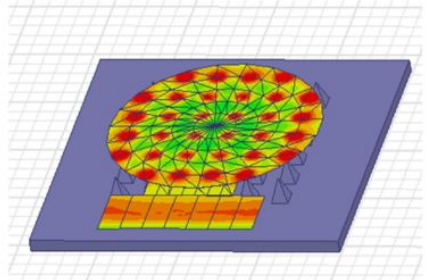

(d) $3 * 3 * 4 \mathrm{~mm}^{3}$

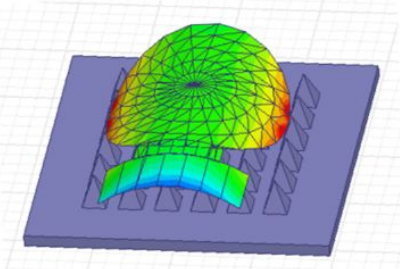

Compression
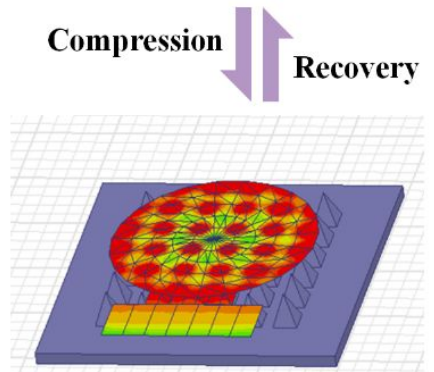

(b)

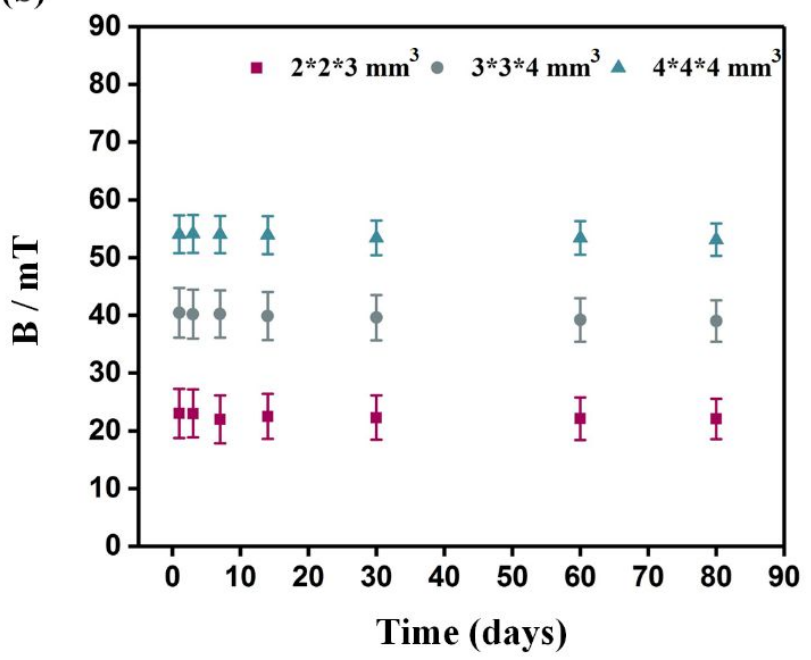

(e)
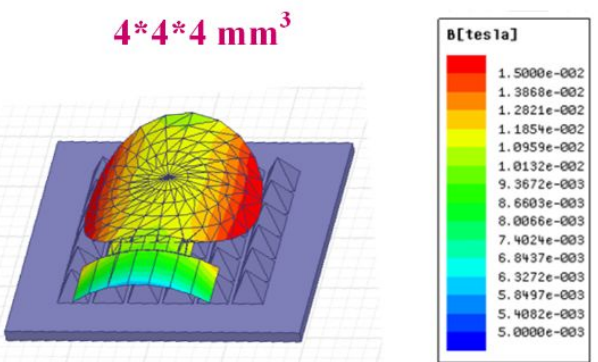

Compression

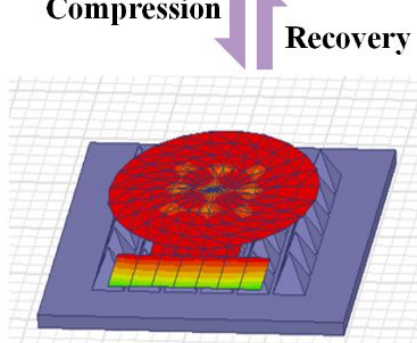

Figure S6. The dependence of magnetic intensity of the magnetic part inside as-fabricated sample on its magnetic pyramid size. (a) Experimental and simulated results of magnetic intensity of magnetic films with different-size magnetic pyramids. (b) The magnetization stability of such three magnetic films over time. 3D calculated distribution of magnetic intensity inside one-cycle liquid metal before and after the same compression via varying in the size of magnetic pyramids from (c) $2 \times 2 \times 3 \mathrm{~mm}$, (d) $3 \times 3 \times 4 \mathrm{~mm}$, to (e) $4 \times 4 \times 4 \mathrm{~mm}$. 
(a)

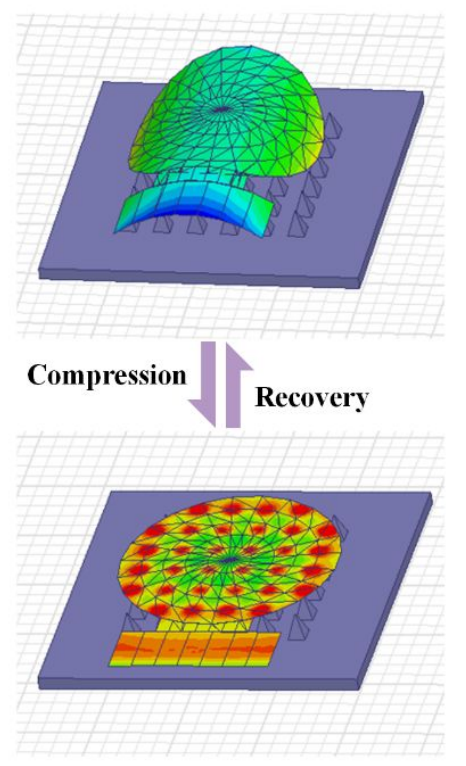

(b) $8 * 8$ array
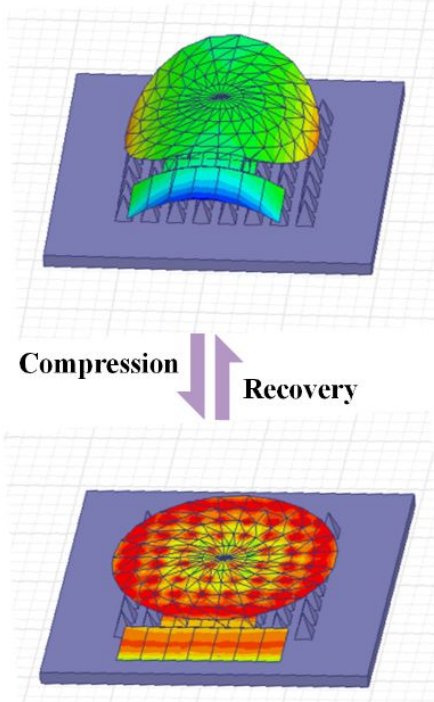

(c) $10 * 10$ array

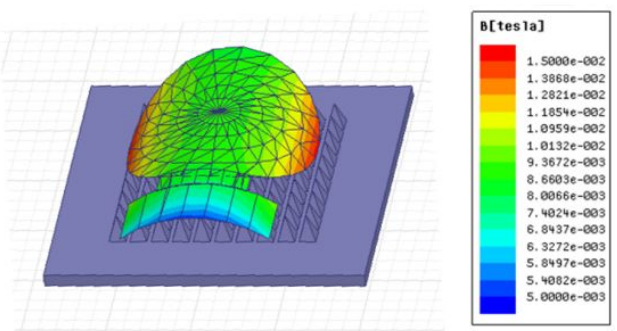

Compression Recovery

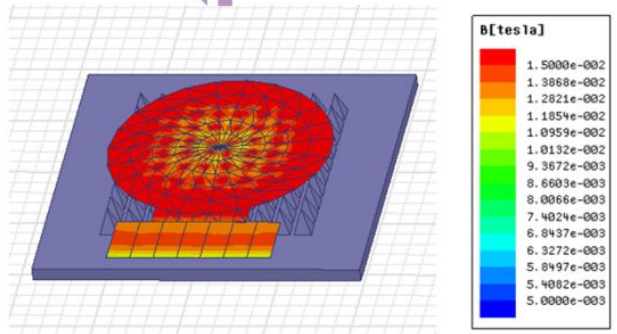

Figure S7. 3D calculated distribution of magnetic intensity inside one-cycle liquid metal before and after the same compression via varying in the number of magnetic pyramids. (a) $6 \times 6$ array, (b) $8 \times 8$ array, and (c) $10 \times 10$ array. Notice: each pyramid-shaped magnetic bulge has the same size.
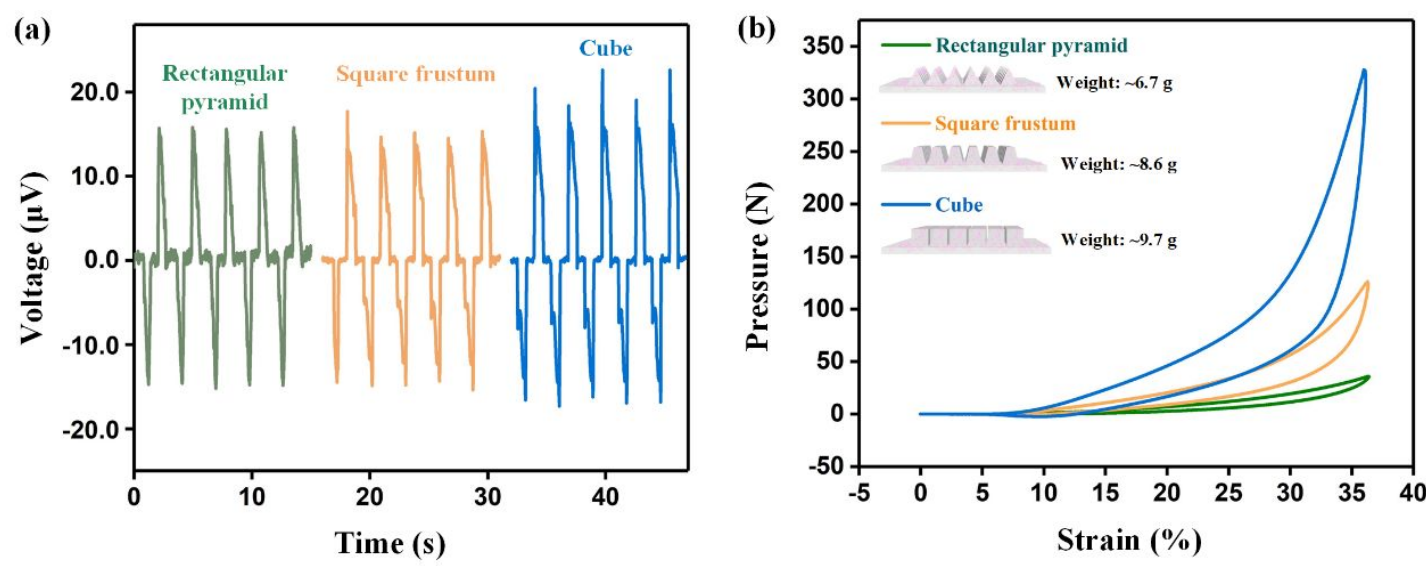

Figure S8. Impact of various geometrical-structured bulges on CSMSs. (a) During the compression test, the open-circuit voltage of CSMSs fabricated with different magnetic films equipped with rectangular pyramid-, square frustum- and cube-shaped bulges. (b) The correlation of pressure and strain curves for the three-typed magnetic films, with their weights. 
(a)

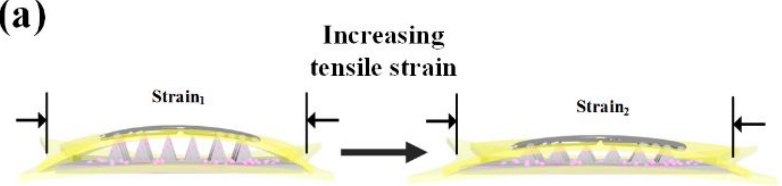

(c)

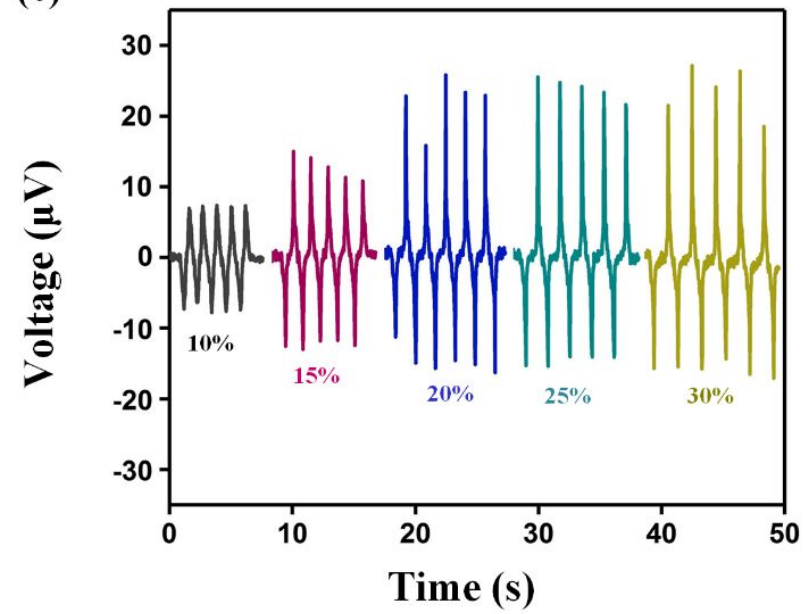

(b)

Increasing tensile speed

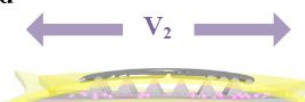

(d)

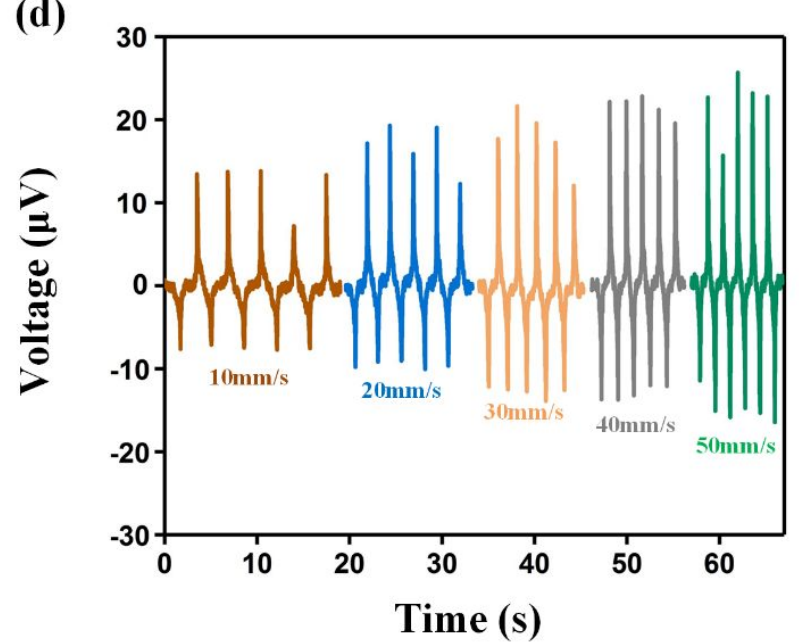

Figure S9. The influence of diverse testing parameters on the electrical performance of the CSMS during stretching process. The output voltage of the same sensor tested in different (c) tensile strain and (d) tensile speed. (a) and (b) are schematic illustrations of (c) and (d), respectively. 

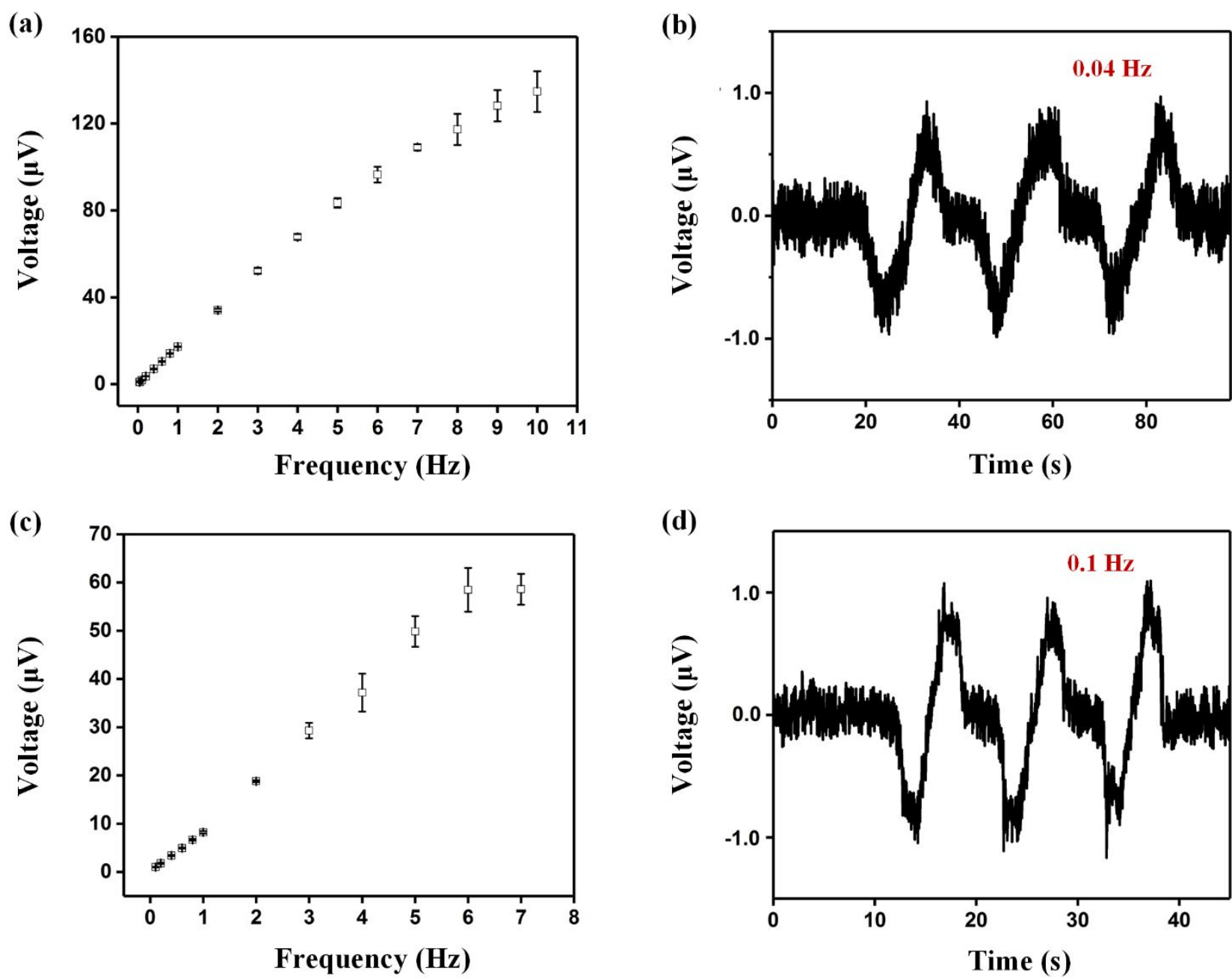

Figure S10. Frequency response of the CSMS. For compressing tests, (a) frequency-voltage correlation of the sensor at $45.5 \%$ compress ratio, and (b) its minimum frequency response of 0.04 Hz. For stretching tests, (c) frequency-voltage correlation of the same sensor at $20 \%$ stretch ratio, and (d) its minimum frequency response of $0.1 \mathrm{~Hz}$. 


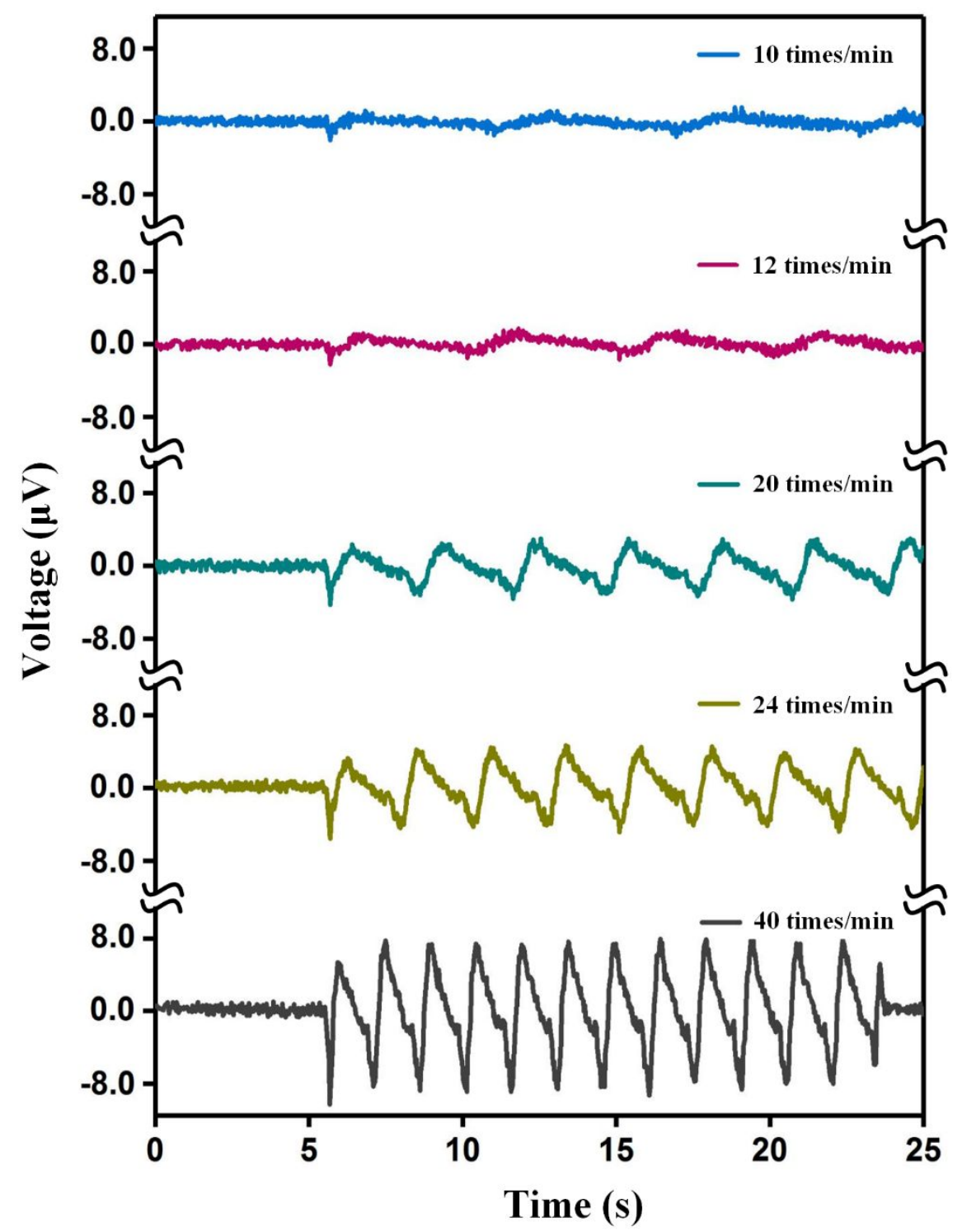

Figure S11. Air-flow driven sensing of CSMS by virtue of a syringe under different injection-exhaust rate. Each injection-exhaust cycle generates two opposite electrical outputs accordingly, meanwhile the output strength increases with the frequency varying from 10 to 40 times/min. 


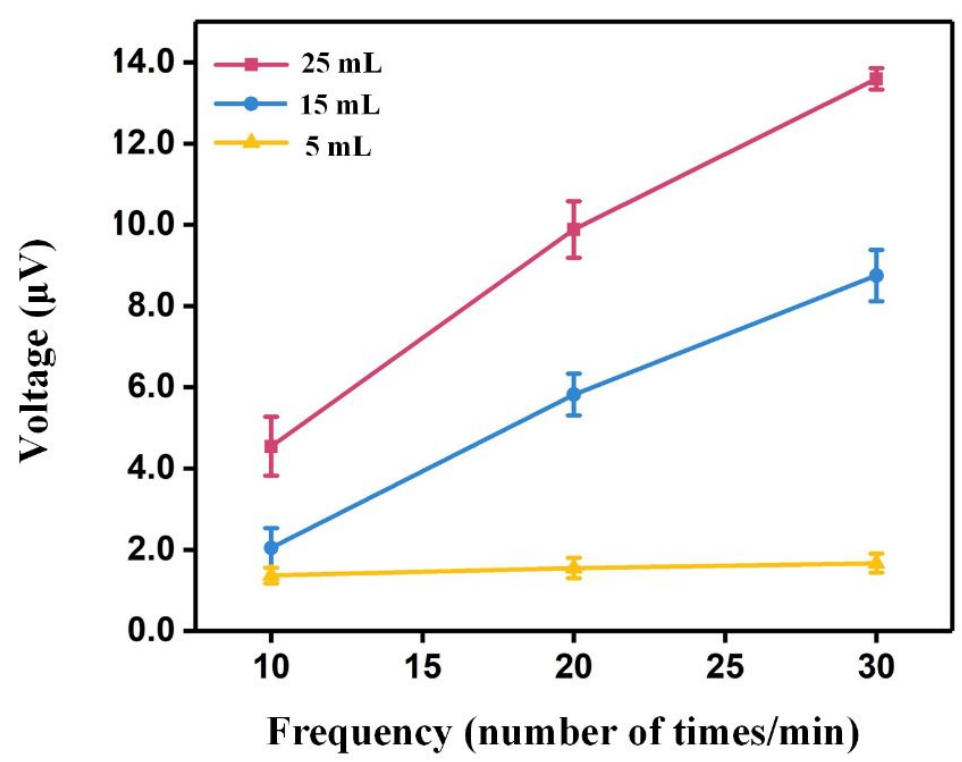

Figure S12. The electrical response of different volumes of exhaled breath under different frequencies. In this case, a syringe was used to exhaust (inject) air with different volumes (5 mL, 15 $\mathrm{mL}, 25 \mathrm{~mL}$ ) to mimic and quantify the different volumes of exhaled breath.

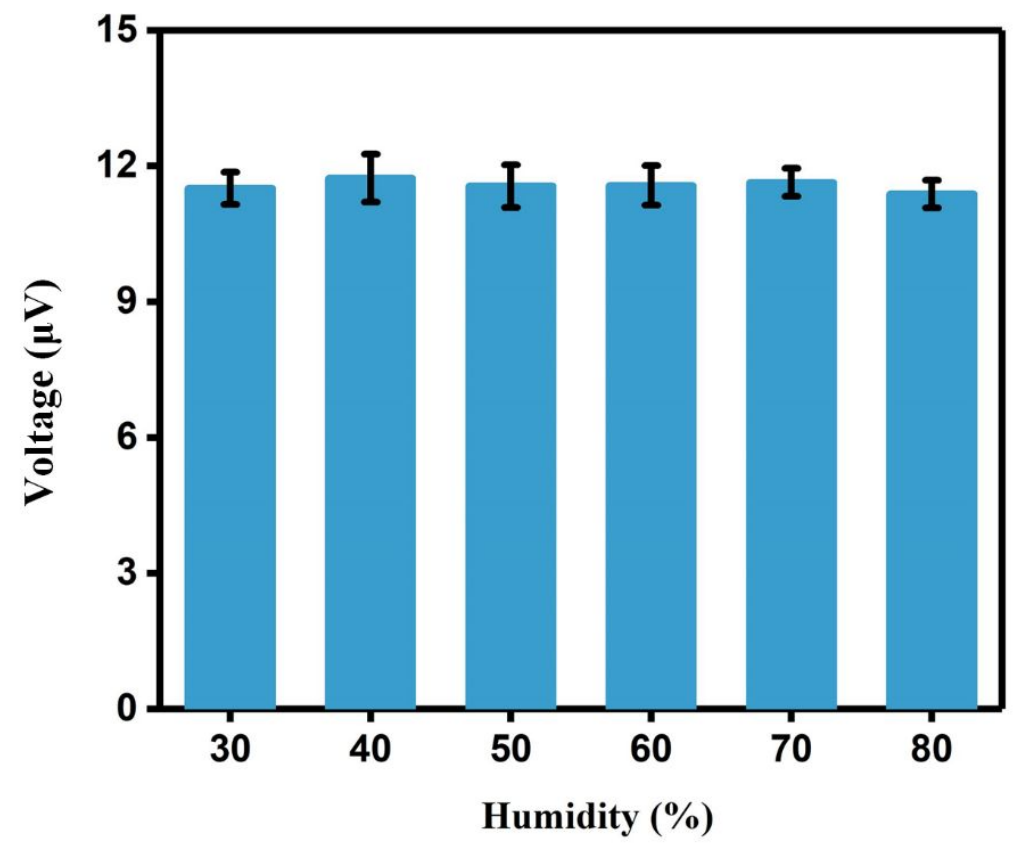

Figure S13. The influence of humidity on the CSMS. The electrical response of the CSMS under various ambient humidity from $30 \%$ to $80 \%$ is basically stable. 


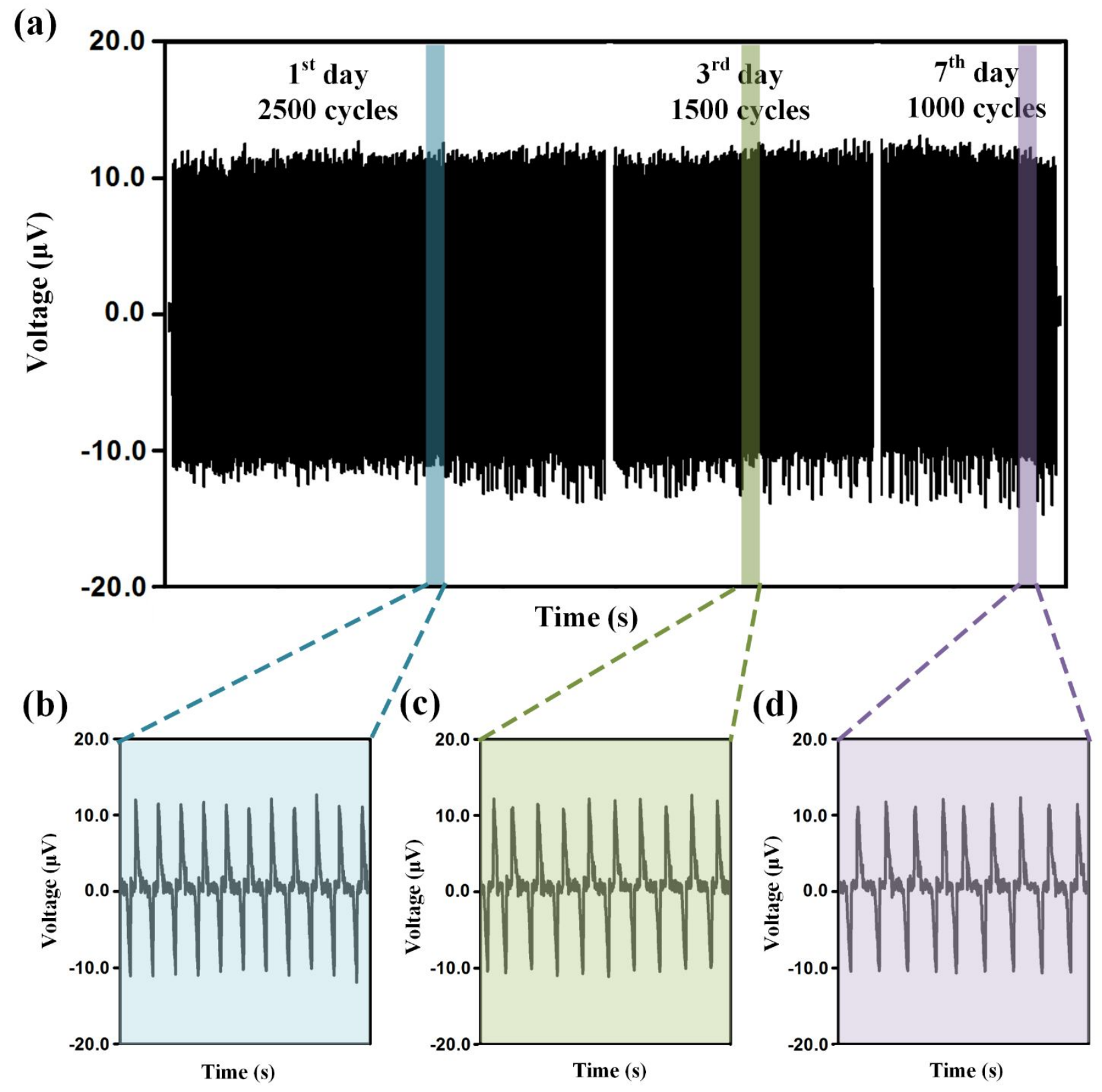

Figure S14. Long-term stability of the CSMS served as a self-powered tactile sensor. (a) Cyclic tests showing the reliability of the magnetoelectric sensor after total 5000 periodic compressing and releasing on the $1^{\text {st }}, 3^{\text {rd }}$ and $7^{\text {th }}$ day. (b), (c) and (d) are the magnified diagrams of (a) in the discontinuously three-day testing. 